\title{
Experimental demonstration of particle acceleration with normal conducting accelerating structure at cryogenic temperature
}

\author{
Mamdouh Nasr@," Emilio Nanni®, Martin Breidenbach@, Stephen Weathersby®, \\ Marco Oriunno@, and Sami Tantawi \\ SLAC National Accelerator Laboratory, 2575 Sand Hill Road, Menlo Park, California 94025, USA
}

(Received 6 March 2021; accepted 9 August 2021; published 13 September 2021)

\begin{abstract}
In this paper, we present an experimental demonstration of the high-gradient operation of an X-band, $11.424 \mathrm{GHz}, 20$-cells linear accelerator (linac) operating at a liquid nitrogen temperature of $77 \mathrm{~K}$. The tested linac was previously processed and tested at room temperature. Low-temperature operation increases the yield strength of the accelerator material and reduces surface resistance, hence a great reduction in cyclic fatigue could be achieved resulting in a large reduction in breakdown rates compared to roomtemperature operation. Furthermore, temperature reduction increases the intrinsic quality factor of the accelerating cavities, and consequently, the shunt impedance leading to increased rf-to-beam efficiency and beam loading capabilities. We verified the enhanced accelerating parameters of the tested accelerator at cryogenic temperature using different measurements including electron beam acceleration up to a gradient of $150 \mathrm{MV} / \mathrm{m}$, corresponding to a peak surface electric field of $375 \mathrm{MV} / \mathrm{m}$. We also measured the breakdown rates in the tested structure showing a reduction of 2 orders of magnitude compared to their values at room temperature for the same accelerating gradient.
\end{abstract}

DOI: 10.1103/PhysRevAccelBeams.24.093201

\section{INTRODUCTION}

The operation of particle accelerators at high-gradient levels is essential for future discovery machines including particle colliders and free-electron lasers [1-3]. It is also essential for advanced medical applications [4,5]. Normal conducting (NC) accelerating structures have been a leading candidate in high-gradient acceleration, with an accelerating gradient above $100 \mathrm{MV} / \mathrm{m}$, for their capability of sustaining higher surface fields compared to their superconducting counterparts [6]. The demanding applications for high-gradient operation and more compact machines derive a continuous international effort to push the boundaries of particle accelerator technology by providing compact accelerating structures with optimized geometries and enhanced accelerating properties.

A limiting factor in the operation of high-gradient accelerating structures is the vacuum arcs on the surface that cause the rf breakdowns which can perturb the acceleration [7]. Consequently, a critical parameter in defining the operation of high-gradient accelerators is

\footnotetext{
*orresponding author. mamdouh@slac.stanford.edu

Published by the American Physical Society under the terms of the Creative Commons Attribution 4.0 International license. Further distribution of this work must maintain attribution to the author(s) and the published article's title, journal citation, and DOI.
}

the rf breakdown rates at the accelerating gradient of interest. Studies have shown that breakdown rates are largely correlated to the peak electric and magnetic fields on the surface. The statistical phenomenon of breakdowns is conjectured to be triggered by crystal defects due to the cyclic fatigue which happens over many cycles. These defects cause surface protrusions seen by the electric field and then cause the breakdown events [8-13]. There has been an effort to minimize the breakdown rates in highgradient accelerators by building accelerator structures from harder materials to reduce the cyclic fatigue on the surface, and thus lower breakdown rates compared to the ones built from softer materials $[14,15]$.

Another approach that was experimentally investigated to enhance the operation of $\mathrm{NC}$ accelerators is the operation at cryogenic temperatures which reduces the surface resistance compared to room-temperature operation. This reduction increases the shunt impedance and internal quality factor of the accelerating cavities, leading to increased rf-to-beam efficiency and beam loading capabilities [16]. The increased rf-to-beam efficiency can increase the overall system efficiency using optimized cryogenic systems. Moreover, low-temperature operation increases hardness [17] reducing the surface deformation and the breakdown rates compared to room-temperature operation for the same accelerating gradient.

Few experiments have investigated the operation of NC accelerating cavities at cryogenic temperatures. In [18], the operation of accelerating cavities at $3 \mathrm{GHz}$ at the liquid 
nitrogen (LN) temperature of $77 \mathrm{~K}$ was investigated. The experiment showed excessive deterioration in the internal quality factor for surface rf magnetic field larger than $1 \mathrm{kGauss}$. The authors concluded that this degradation is caused by the high rf magnetic field on the surface. Other studies were performed at low-gradient levels, below $50 \mathrm{MV} / \mathrm{m}[19,20]$. These experiments verified the internal quality factor of the tested cavities at cryogenic temperatures. However, they did not demonstrate high-gradient operation nor study the breakdown rates in the tested structures. An investigation performed by CERN studied the breakdown rates for a set of cavities with frequencies between 21 and $35 \mathrm{GHz}$ at operating temperatures between 100 and $500 \mathrm{~K}$ [21]. The study showed no dependence of the maximum obtainable field on the temperature of operation.

A recent experimental effort aimed at providing a more complete picture of the operation of high-gradient accelerating cavities at cryogenic temperatures [22]. The authors performed an experimental investigation on a single-cell accelerating structure at $11.424 \mathrm{GHz}$ and $45 \mathrm{~K}$. They reported the higher operation field at the same breakdown rate at cryogenic temperatures compared to room-temperature operation. The room-temperature data were obtained from previous studies with similar structures. Since the breakdown rate is a steep-increasing function of field, it leads to the idea of the reduced breakdown rates at cryogenic temperatures compared to room-temperature operation. This reduction enabled reaching an accelerating gradient of $250 \mathrm{MV} / \mathrm{m}$. They also reported a time-dependent degradation in the internal quality factor of the accelerating cavity for accelerating gradients higher than $150 \mathrm{MV} / \mathrm{m}$ [23]. The time-dependent degradation was reported to be due to elevated levels of field emission and dark current. The experiments observed the onset of breakdowns at an accelerating gradient of $250 \mathrm{MV} / \mathrm{m}$, but were unable to characterize the breakdown probability's dependence on accelerating gradient with the collected statistics.

The experimental effort in [18-20,22,23] showed a large promise in the operation of $\mathrm{NC}$ accelerators at cryogenic temperatures for enhanced shunt impedance and reduced breakdown rates. This effort was performed, however, for single-cell testing and/or low-gradient operation, below $50 \mathrm{MV} / \mathrm{m}$. Further, the lack of defined slopes for the breakdown rates versus the accelerating gradient at cryogenic temperature does not provide a clear understanding of the statistical behavior of breakdown rates. Most importantly, these experiments did not attempt to demonstrate particle acceleration with the tested structures. To the best of our knowledge, there has never been an experimental demonstration of the operation of multicell NC accelerating structures at cryogenic temperature and high-gradient levels.

In our work, we developed a practical approach for operating high-gradient linacs at cryogenic temperature. This development is a necessary milestone in the road of adapting cryogenic-NC accelerators in many practical applications, including future discovery machines. Practicality and economic aspects are thus important. We decided to operate at the cryogenic temperature of $\mathrm{LN}(77 \mathrm{~K})$. Operating with the LN cooling system results in much-reduced cost compared to the complicated setup required for operation with liquid helium, while still largely enhancing the shunt impedance and beam-loading capabilities of $\mathrm{NC}$ accelerator structures compared to room-temperature operation. We verified the accelerating parameters using many approaches including energy-gain measurements with an electron beam. We also performed an extensive study on the breakdown rates for the same accelerator structure at room and cryogenic temperatures. The tested linac structure utilizes a distributedcoupling power-feeding topology which permits more degrees of freedom for the optimization of individual cavity shapes in comparison with conventional linacs [24]. Combining the enhanced shunt impedance using the distributed-coupling technology with the boosted performance at cryogenic temperature is capable of providing new frontiers in $\mathrm{NC}$ accelerating structures at high-gradient operation.

\section{THE THEORY OF ANOMALOUS SKIN EFFECT}

The rf surface resistance of good conductors at cryogenic temperatures is well studied and explained using the theory of anomalous skin effect (ASE) [25]. At low temperatures and high frequencies, the mean-free path of electrons, $l$, becomes comparable or larger than the classical skin depth, $\delta$, for good conductors. In this case, electrons that contribute the most to the current are the ones that spend their entire mean-free path in the skin depth, and the surface resistance saturates to a higher value than the one predicted by the classical skin depth.

To calculate the surface resistance, including the ASE, first, we can calculate the conductivity of the metal at a certain operating temperature, $T$, using the BlochGrüneisen formula [26] as

$$
\frac{1}{\sigma_{c}(T)}=\rho_{c}(T)=\frac{C}{M \theta}\left(\frac{T}{\theta}\right)^{5} \int_{0}^{\theta / T} \frac{z^{5} e^{z}}{\left(e^{z}-1\right)} d z+\psi,
$$

where $\rho_{c}(T)$ is the electrical resistivity of the metal, $\theta$ is the electrical resistivity Debye temperature, $M$ the atomic weight, $C$ is a constant set to achieve the known intrinsic resistivity of the metal at $293 \mathrm{~K}$ and $\psi$ is a correction constant which we added. For copper, $\rho(293 \mathrm{~K})=1.78 \times$ $10^{-8} \Omega \mathrm{m}$ and we calculated $C=3.123 \times 10^{-7}$.

We can note that, for $\psi=0$, the equation predicts zero resistivity at $0 \mathrm{~K}$ which is practically impossible due to the impurities in the metal. A common parameter used to indicate the purity of the metal is the residual-resistivity ratio (RRR), defined as $\mathrm{RRR}=\rho_{c}(293 \mathrm{~K}) / \rho_{c}(4 \mathrm{~K})$. We added $\psi$ in (1) to achieve the RRR value of the used metal. For the used copper material in our structure, $R R R=400$ was achieved with $\psi=0.046 \times 10^{-8}$. Note that the RRR of 
the used copper has a minor effect on the conductivity at $\mathrm{LN}$ temperature of $77 \mathrm{~K}$, but becomes more dominant at lower temperatures.

The mean-free path for free electrons is related to the conductivity of the metal using the Drude model of metals [27] as

$$
\sigma_{c}=\frac{\omega_{p}^{2} l}{Z_{0} c v_{f}},
$$

where $c$ is the speed of light, $Z_{0}$ is the free-space impedance, $Z_{0}=120 \pi \Omega, \omega_{p}$ is the plasma frequency for the metal, and $v_{f}$ is the Fermi velocity. For copper, $\omega_{p}=1.7 \times 10^{16} \mathrm{rad} / \mathrm{s}$ and $v_{f}=1.6 \times 10^{6} \mathrm{~m} / \mathrm{s}$. Also, the skin depth is expressed in terms of conductivity as

$$
\delta(\omega)=\sqrt{\frac{2 c}{Z_{0} \omega \sigma_{c}}}
$$

where $\omega$ is the operating frequency. Consequently, knowing the conductivity, we can calculate the mean-free path and skin depth using Eqs. (2) and (3), respectively.

Finally, the surface impedance, $Z_{s}$, can be calculated using the equations for the anomalous skin effect in the simplified form presented in [28] as

$$
Z_{s}^{*}(\omega)=-\frac{i \omega l}{c F(\omega)} Z_{0},
$$

where the function $F$ is defined by

$$
F(\omega)=-\frac{1}{\pi} u \int_{0}^{\infty} \ln \left(1+\frac{\eta+\xi \chi(t)}{t^{2}}\right),
$$

and

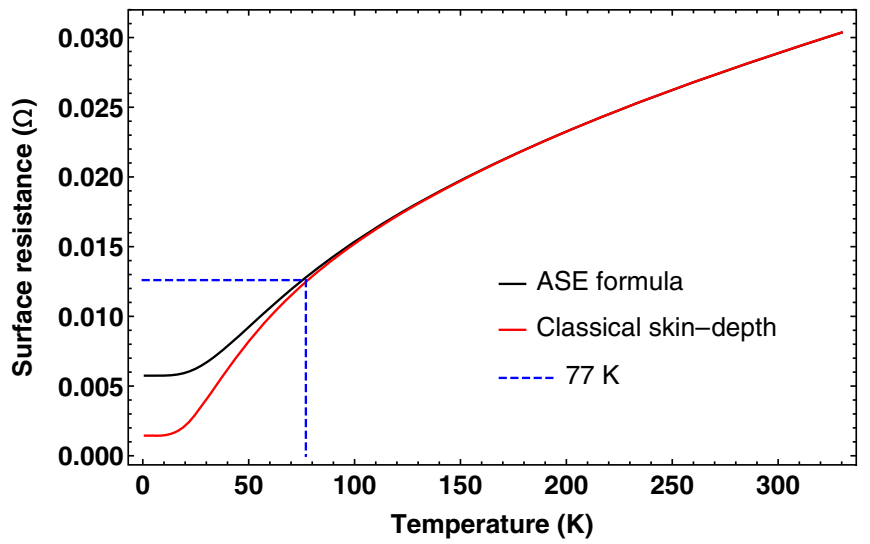

FIG. 1. A plot of the surface resistance for copper with RRR = 400 versus temperature at $11.424 \mathrm{GHz}$. At low temperatures, the surface resistance, predicted by ASE, saturates to higher values than the ones calculated from the classical skin depth. Operating at $77 \mathrm{~K}$ results in a reduction in the surface resistance of a factor of 2.25 compared to $300 \mathrm{~K}$. $u=1+i \frac{\omega l}{v_{f}}, \quad \eta=-\frac{\omega^{2} l^{2}}{c^{2} u^{2}}, \quad \alpha=\frac{3}{2}\left(\frac{l}{\delta(\omega)}\right)^{2}$,

$\xi=i \alpha u^{-3}, \quad \chi(t)=2 t^{-3}\left[\left(1+t^{2}\right) \arctan (t)-t\right]$.

Figure 1 shows the surface resistance for copper with $\mathrm{RRR}=400$, the nominal value used in our structures, versus temperature at $11.424 \mathrm{GHz}$. At low temperatures, the surface resistance predicted by the ASE saturates to higher values than the ones calculated from the classical skin depth. From our calculations, operating at $77 \mathrm{~K}$ results in a reduction of a factor of 2.25 in surface resistance compared to $300 \mathrm{~K}$ operation.

\section{EXPERIMENTAL SETUP AND TESTING RESULTS}

In our cryogenic experiment, we tested an X-band, 11.424 GHz, 20-cells, standing-wave linac structure which has been previously processed and tested at room temperature. The operating gradient of the structure was successfully verified up to a gradient of $140 \mathrm{MV} / \mathrm{m}$ at $300 \mathrm{~K}$ [24]. Consequently, we can compare the performance of the same accelerator structure at room and cryogenic temperatures. Table I shows the distributed-coupling linac parameters at 300 and $77 \mathrm{~K}$. Note that every part of the system shrinks proportionally, including the distance between accelerating cells. So, as long as the structure is operating at the shifted resonance frequency, the $\pi$-mode is properly maintained resulting in keeping synchronous between beam and rf phase. Moreover, the external quality factor, $Q_{\text {ext }}$, remains constant.

We modified the existing setup at the X-band Test Accelerator (XTA) station at the Next Linear Collider Test Accelerator (NLCTA) facility at SLAC [29] to accommodate high-power experiments at cryogenic temperatures. The experimental setup is shown in Fig. 2(a). XTA is divided into two stations, referenced in Fig. 2(a) as stations 1 and 2, and each one is fed with an independent $\mathrm{rf}$ source. The first station is used to generate the electron

TABLE I. Summary of the accelerating parameters of the distributed-coupling accelerating structure at 300 and $77 \mathrm{~K}$. The peak fields are calculated for an average accelerating gradient of $100 \mathrm{MV} / \mathrm{m}$.

\begin{tabular}{lcc}
\hline \hline Parameter & $300 \mathrm{~K}$ & $77 \mathrm{~K}$ \\
\hline Frequency $(\mathrm{GHz})$ & 11.402 & 11.438 \\
$Q_{0}$ & 10000 & 22500 \\
$Q_{\text {ext }}$ & 10000 & 10000 \\
Shunt impedance $(\mathrm{M} \Omega / \mathrm{m})$ & 155 & 349 \\
Peak surface E $(\mathrm{MV} / \mathrm{m})$ & 250 & 250 \\
Peak surface H $(\mathrm{MA} / \mathrm{m})$ & 0.575 & 0.575 \\
Steady state rf power $(\mathrm{MW})$ & 17 & 9 \\
Iris diameter (mm) & 2.6 & 2.6 \\
Length $(\mathrm{cm})$ & 26 & 26 \\
\hline \hline
\end{tabular}




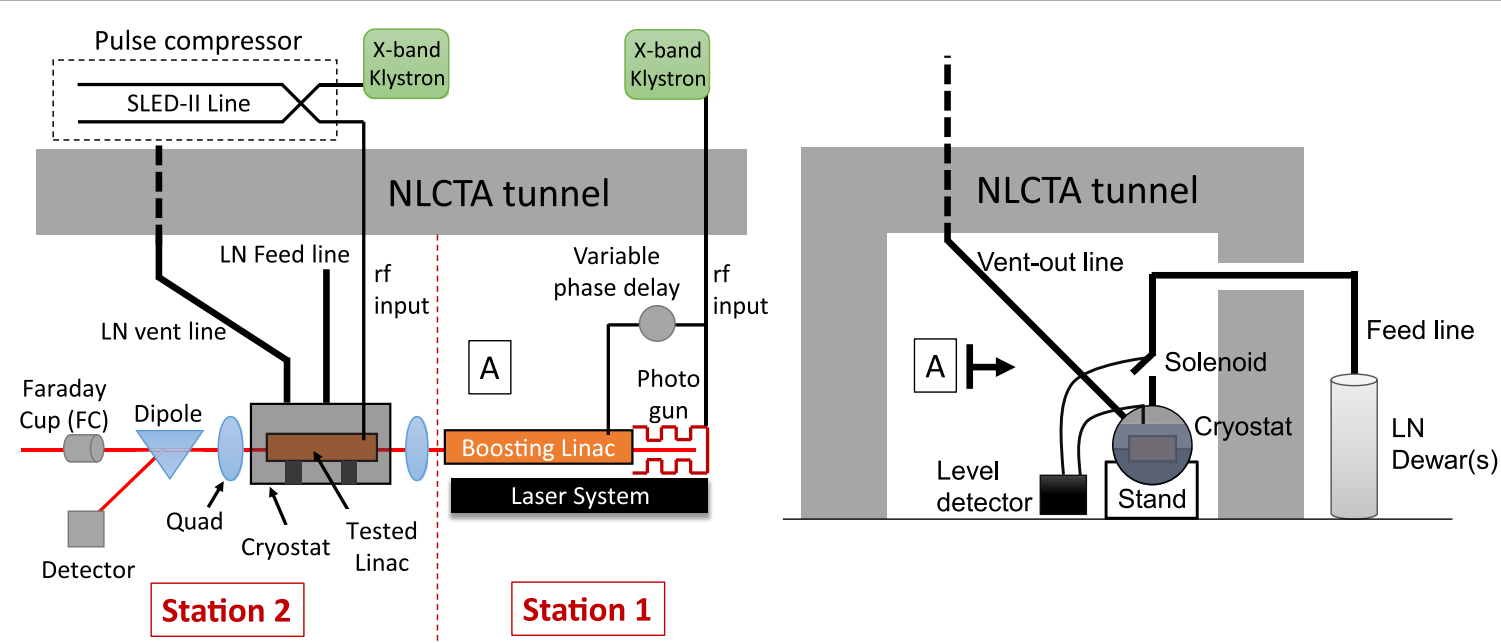

(a)

(b)

FIG. 2. (a) The experimental setup for accelerator testing at the XTA facility at SLAC. XTA is divided into two stations, referenced to as station 1 and station 2. The first station generates the electron beam used in the experiment, and the tested accelerator is installed in the second station. (b) A foam-insulated stainless-steel line delivers LN from Dewars outside the tunnel to the cryostat where the tested linac is setting inside, through a hole in the tunnel wall. A level detector is inserted inside the cryostat and provides a control signal to a solenoid that controls the flow of the LN in the feedline. A vent-out line transports the boiled LN outside the tunnel.

beam from an X-band photoinjector and then accelerate the beam to about $46 \mathrm{MeV}$ using an NLC-style X-band, $1 \mathrm{~m}$ long, accelerator [30]. The second station feeds the distributed-coupling linac, which is installed after the station 1 linac.

An X-band klystron feeds the linac under testing with rf power. The peak rf power of the klystron is $40 \mathrm{MW}$ and we installed a pulse compressor (multimoded SLED-II [31]) after the klystron to boost the rf power above the klystron limit. Quadrupoles and steers are used for beam focusing and trajectory adjustment, a spectrometer is placed after the distributed-coupling linac to measure the beam energy, and a Faraday cup (FC) is installed downstream for charge measurements. The breakdown events were identified by detecting the dark-current spikes from the linac at the FC.

Figure 2(b) gives an illustration of the used cooling system for our cryogenic experiment. We drilled a hole through the tunnel wall, perpendicular to the beam axis. A foam-insulated stainless-steel line delivers LN from Dewars outside the tunnel to the cryostat, inside which the distributed-coupling linac is sitting. A level detector detects the level of the LN inside the cryostat and provides a control signal to a solenoid that controls the flow of the LN in the feedline. A vent-out line transports the boiled LN outside the tunnel.

The used cryostat is a simple cylindrical stainless-steel container with insulation foam from the outside. The linac structure is sitting inside the cryostat on two half-circular supports with a rectangular opening for the structure to sit inside. The cryostat and the linac share the same beam axis and there are two transport tubes under vacuum from both sides of the linac to the cryostat walls, perpendicular to the beam line. The cryostat has an opening for each of the LN and $\mathrm{rf}$ feedlines, in addition to small openings for the $\mathrm{LN}$ level detector and vent-out line connections. The linac structure shares vacuum with the beam line, and the cryostat is not vacuum insulated.

\section{A. Cold test of the linac structure at cryogenic temperature}

Before installing the cryostat into the beam line, we performed a cold test of our accelerator structure at cryogenic temperature. We installed the accelerator structure, under vacuum, inside the cryostat and used an LN

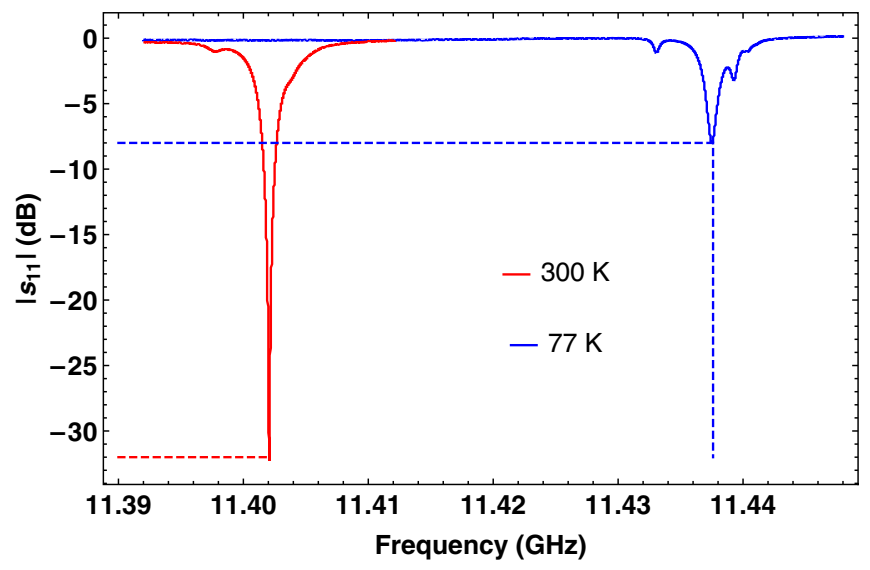

FIG. 3. The reflection coefficient at the rf input to the accelerator at 300 and $77 \mathrm{~K}$. As the accelerator structure cools down, it shrinks leading to a shift of the resonance frequency of $36 \mathrm{MHz}$. The tested linac is designed for critical coupling at $300 \mathrm{~K},\left|s_{11}\right|=$ $-32 \mathrm{~dB}$ at resonance, and becomes overcoupled at $77 \mathrm{~K},\left|s_{11}\right|=$ $-8 \mathrm{~dB}$ at resonance, because of the reduced surface resistance which increases the intrinsic quality factor of the accelerating cavity while the external one remains constant. 


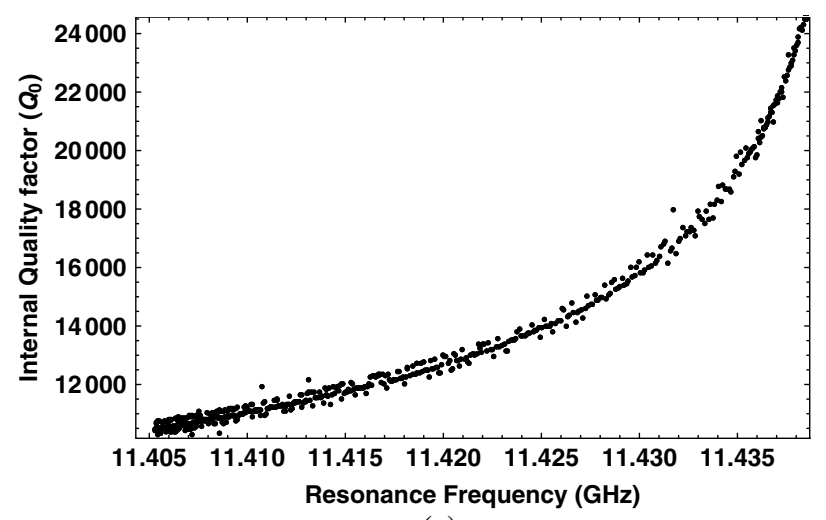

(a)

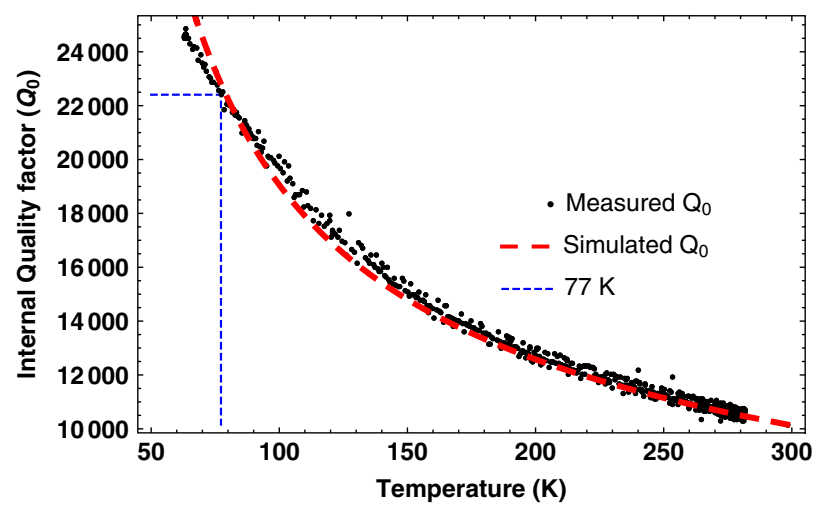

(b)

FIG. 4. (a) The measured intrinsic quality factor of the accelerating structure versus the resonance frequency of the structure. The measured values are obtained using Q-circle fitting of the measured reflection coefficient at the rf input port. (b) We compared the measured values of the internal quality (black dots) with our simulations (dashed line). In our simulations, the temperature at each point is used to calculate the surface resistance using the theory of ASE and simulate the intrinsic quality factor.

Dewar to supply LN directly into the cryostat. A vector network analyzer (VNA) was connected to the rf input of the structure, through a vacuum window, to measure the reflection coefficient at the rf input port to the accelerator.
Figure 3 shows the reflection coefficient at the rf input to the accelerator at 300 and $77 \mathrm{~K}$. As the accelerator structure cools down, it shrinks, and thus the resonance frequency shifts to a higher value. We measured a resonance shift of $36 \mathrm{MHz}$ from 300 to $77 \mathrm{~K}$ which is in good agreement with the calculated value using the thermal expansion of copper [32]. The distributed-coupling linac is designed for critical coupling at $300 \mathrm{~K}$ and becomes overcoupled at $77 \mathrm{~K}$ because of the reduced surface resistance, which increases the internal quality factor, $Q_{0}$, of the accelerating cavity. We should emphasize that the increase of the coupling coefficient, $\beta=Q_{0} / Q_{\mathrm{ext}}$, for the accelerating structure from 1 at $300 \mathrm{~K}$ to 2.25 at $77 \mathrm{~K}$ represents a large increase in the beam-loading capability (allowing for larger beam charge) for the same accelerator structure just by cooling it down to cryogenic temperature.

We carried out another test to measure the change in the quality factor and resonance frequency for the accelerator structure versus temperature. We supplied LN into the cryostat until the structure temperature stabilized. We then stopped the LN supply and measured the reflection coefficient as the structure warms up to room temperature. Figure 4(a) shows the measured internal quality factor of the accelerating structure versus resonance frequency from 300 to $67 \mathrm{~K}$. The measured values of the internal quality factor were obtained using Q-circle fitting of the measured reflection coefficient at the rf input port [33].

We compared the measured values of the internal quality with our simulations, represented by the dashed line in Fig. 4(b). In the simulations, we calculated the temperature at each point from the measured shift of the resonance frequency compared to its value at room temperature, and the thermal expansion coefficient of copper [32]. We then used the temperature value to calculate the surface resistance using the theory of ASE, previously presented in Fig. 1. The obtained surface resistance is used to simulate the internal quality factor of the accelerator structure. Figure 4(b) shows good agreement between the measured and simulated values. The lowest measured temperature is $67 \mathrm{~K}$. However, for high-power operation,

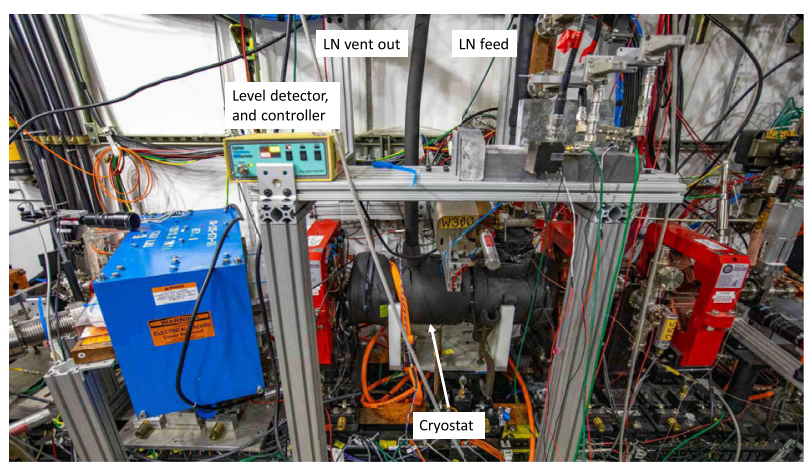

(a)

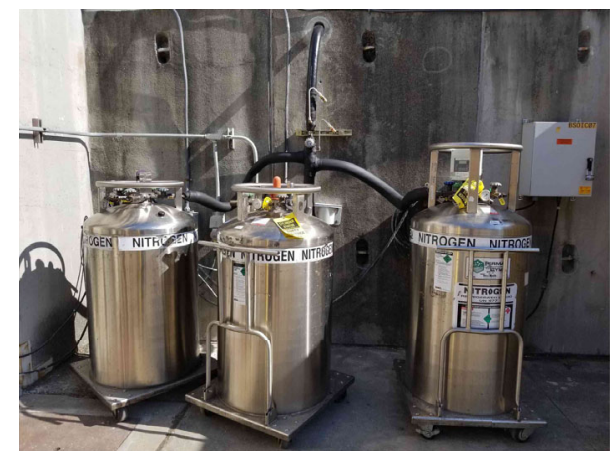

(b)

FIG. 5. (a) A picture of the installed cryostat in the XTA beam line and (b) the LN feeding system outside the tunnel. 


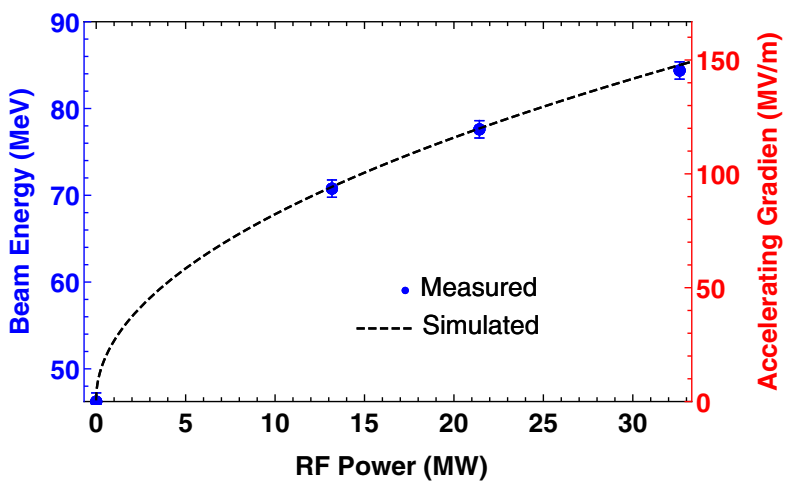

FIG. 6. The measured energy gain of an electron beam moving down the axis of the distributed-coupling linac for a compressed 200 ns square rf pulse with an input power of 13-33 MW, achieving an accelerating gradient of $100-150 \mathrm{MV} / \mathrm{m}$ and a peak surface electric field of $250-375 \mathrm{MV} / \mathrm{m}$. Blue dots and the dashed line are measured and simulated values, respectively. Simulations used the measured input rf pulse and the cavity model at $77 \mathrm{~K}$. the temperature stabilizes at $77 \mathrm{~K}$ with an internal quality factor of 22500.

\section{B. High-power experiment at cryogenic temperature}

After verifying the quality factor of our accelerating structure at low-level rf, we installed the cryostat in the XTA beam line (Fig. 5). The goal of our experiment is to verify the accelerating parameters of our structure and to collect breakdown statistics for high-power operation at $77 \mathrm{~K}$. We measured the energy gain of an electron beam moving down the axis of the tested linac and compared the results with our simulations using the measured input pulse to the structure and the cavity model, substituting with the accelerating parameters from Table I at $77 \mathrm{~K}$ [34].

The electron beam is formed from a single bunch with a bunch length of around $400 \mathrm{fs}$ and a charge of $100 \mathrm{pC}$. The tested structure operated at $11.438 \mathrm{GHz}$, while the rf gun and a $1 \mathrm{~m}$ long accelerator that followed the gun operated at 11.424 GHz. This was possible because the electron gun and the $1 \mathrm{~m}$ long conventional accelerator are fed by one klystron, while the tested accelerator structure is operated

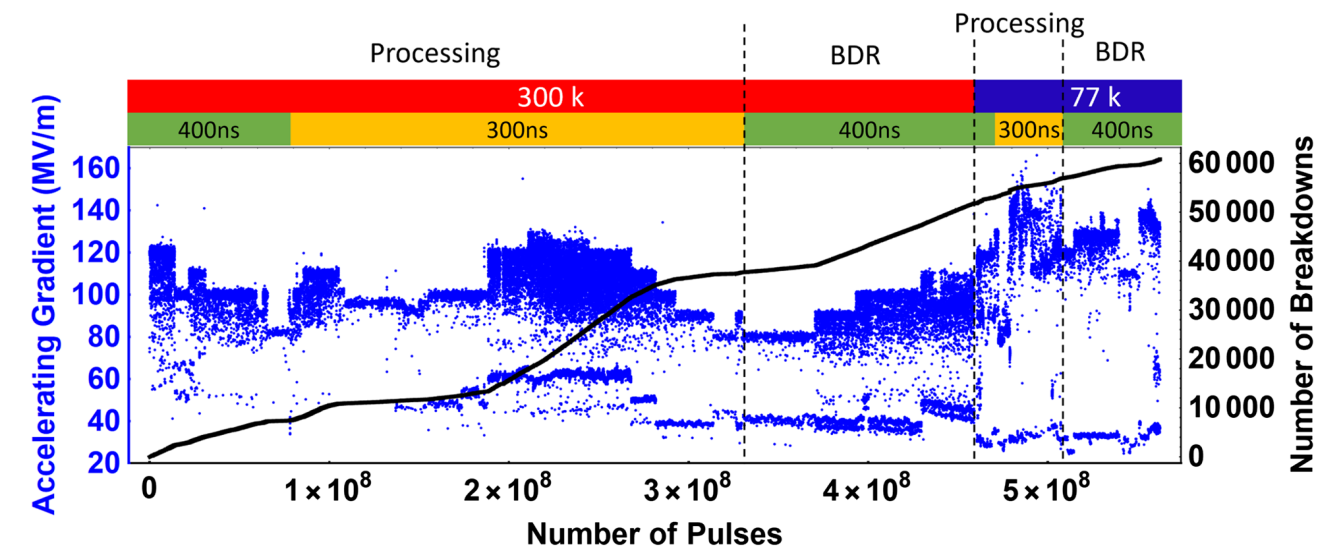

(a)

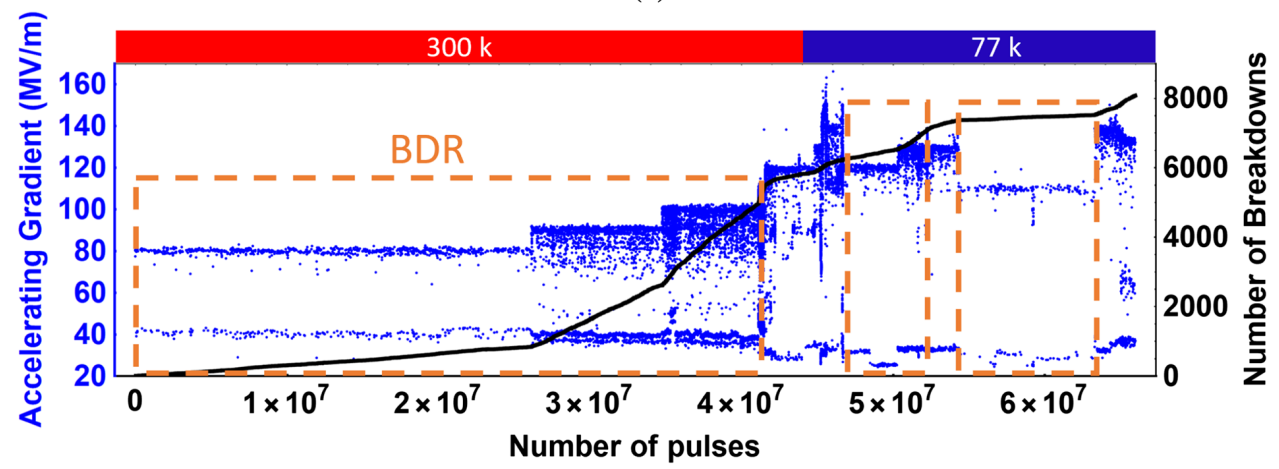

(b)

FIG. 7. (a) The processing history of the distributed-coupling linac at 300 and $77 \mathrm{~K}$ with the total number of pulses and breakdowns. The structure was tested using $400 \mathrm{~ns}$ stepped pulses with $200 \mathrm{~ns}$ flat gradient and $300 \mathrm{~ns}$ pulses with $100 \mathrm{~ns}$ flat gradient. (b) A zoom-in of the collected breakdown statistics at 300 and $77 \mathrm{~K}$ and different gradient levels. In the second plot, we discarded the periods of ramping up to the target gradient, following a breakdown event, in our calculations of the number of pulses and breakdowns. The BDR are collected for $400 \mathrm{~ns}$ stepped pulses with $200 \mathrm{~ns}$ flat gradient. 
with another klystron as shown in Fig. 2. The two klystrons are fed by the same synchronous source at $11.424 \mathrm{GHz}$, but we added a phase chirp to the klystron feeding the tested accelerator to raise the frequency to 11.438 . This allowed us to control the timing between injected bunch and the rf in the tested accelerator precisely. Operating the tested linac at a slightly higher frequency resulted in an overall phase slippage of $4.37^{\circ}$ across the whole length of the accelerator structure $(\sim 26 \mathrm{~cm})$.

We performed a set of measurements using a compressed 200 ns square rf pulse with an input power of 13-33 MW achieving an accelerating gradient from $100-150 \mathrm{MV} / \mathrm{m}$, and a peak surface electric field of $250-375 \mathrm{MV} / \mathrm{m}$. Figure 6 shows the measured energy gain versus input rf power showing very good agreement between the measurements and simulations, less than $3 \%$ deviation.

In our experiment, we studied the correlation between the breakdown rates (BDR) and the operating temperature of the tested accelerator structure. Figure 7(a) shows the processing history of the structure at room and cryogenic temperatures. The curve displays the total number of observed breakdowns and counted pulses. The plot also highlights the processing and the breakdown test periods. The structure processing spans over $5 \times 10^{8}$ pulses with $10^{8}$ pulses at $77 \mathrm{~K}$.

In our calculations of the breakdown rates, we eliminated any observed breakdowns that result from system failures in the SLED line, klystron, or any other system components. We also eliminated the periods of ramping up to the target gradient. As a result, the reported breakdown rate at a specific gradient level is calculated using the counted breakdowns and pulses while the structure is settling at this gradient level. Figure 7(b) shows a zoom-in with the counted breakdowns and pulses for the recorded gradient levels at room and cryogenic temperatures. The breakdown rates were collected up to a gradient level of 110 and $130 \mathrm{MV} / \mathrm{m}$ at 300 and $77 \mathrm{~K}$, respectively. We attempted to collect breakdown rates above $130 \mathrm{MV} / \mathrm{m}$ at $77 \mathrm{~K}$, but encountered temperature instability in the structure.

Figure 8 shows the measured forward and reflected pulses at the input of the accelerator structure at a gradient of 95 and $120 \mathrm{MV} / \mathrm{m}$ for 300 and $77 \mathrm{~K}$ operation, respectively. The figure also shows the simulated gradient using the cavity model and the good agreement between the measured and simulated reflected waveform.

Figure 9 shows the collected breakdown rates, with the fitted slope, for the tested accelerator structure at $77 \mathrm{~K}$ with an accelerating gradient of $110-130 \mathrm{MV} / \mathrm{m}$. The breakdown rates are compared with the ones for the same accelerator structure at $300 \mathrm{~K}$. For both operating temperatures, the data were collected for a 400 ns stepped pulse with a flat gradient of $200 \mathrm{~ns}$ and $60 \mathrm{~Hz}$ repetition rate. The breakdown statistics at each gradient level were collected over more than $3 \times 10^{6}$ pulses. The only exception is the collected statistics at $110 \mathrm{MV} / \mathrm{m}$ for $300 \mathrm{~K}$ where the data was collected over $4 \times 10^{5}$ pulses.
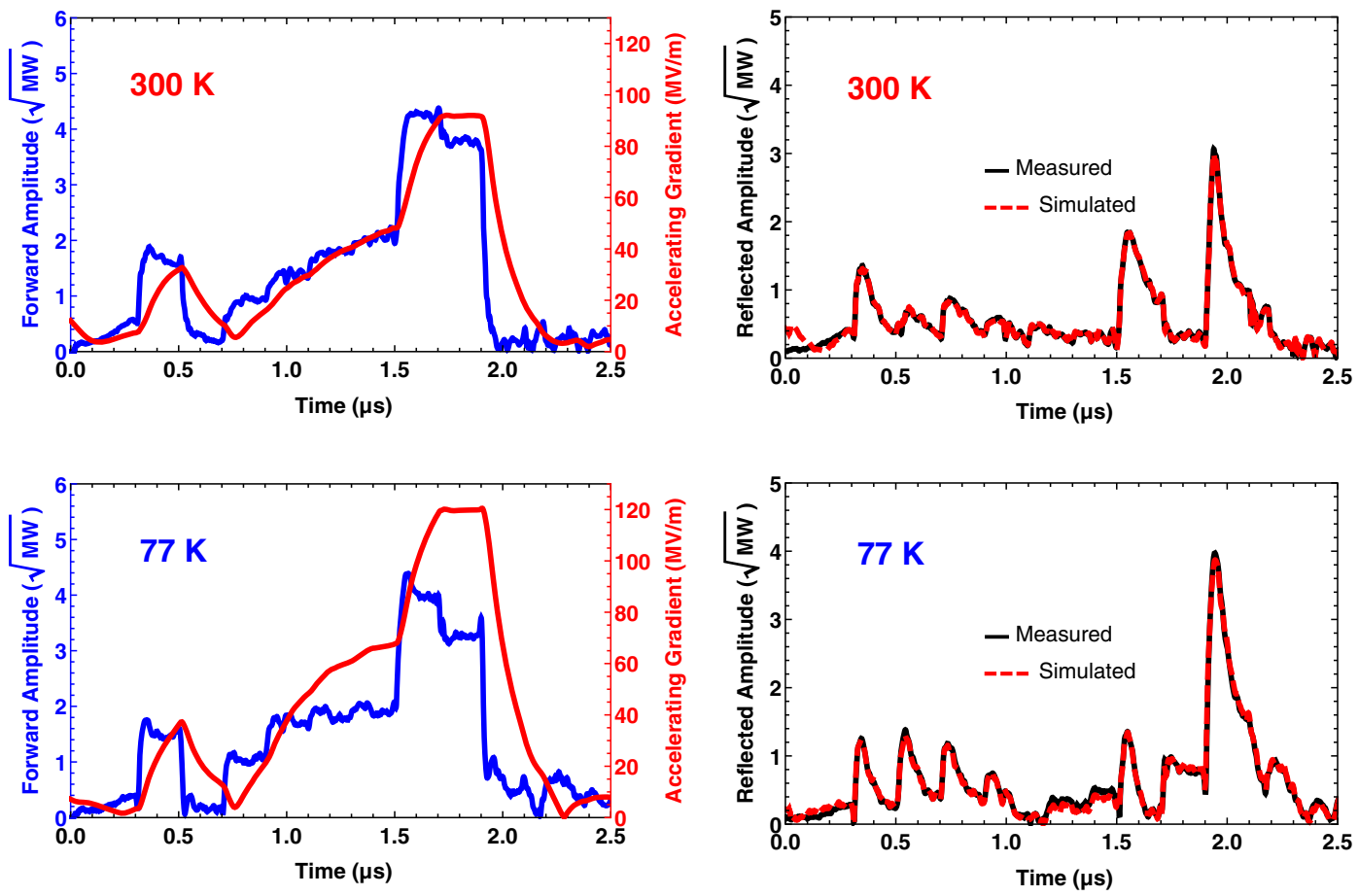

FIG. 8. The measured forward and reflected pulses at the input of the accelerator structure at a gradient of 95 and $120 \mathrm{MV} / \mathrm{m}$ for 300 and $77 \mathrm{~K}$ operation, respectively. The figure also shows the simulated gradient using the cavity model and the good agreement between measured and simulated reflected waveform. 


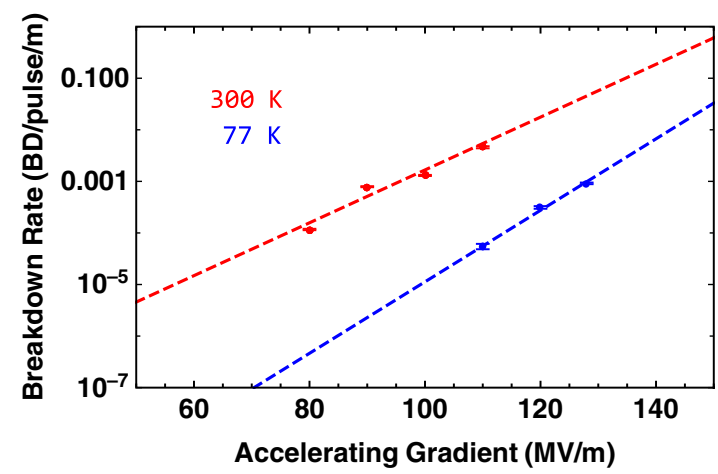

FIG. 9. The collected breakdown rates with the fitted slope of the distributed-coupling accelerator structure at 300 and $77 \mathrm{~K}$. The breakdown rates at $300 \mathrm{~K}$ are obtained from our previous testing of the same accelerator structure at room temperature. For both operating temperatures, the data were collected for a $400 \mathrm{~ns}$ stepped pulse with a flat gradient of $200 \mathrm{~ns}$. The results show a reduction by 2 orders of magnitude, $\times \frac{1}{100}$, in breakdown rates at the same gradient levels from 300 to $77 \mathrm{~K}$ operation.

The results show a reduction of 2 orders of magnitude, $\times \frac{1}{100}$, in breakdown rates at the same gradient levels from 300 to $77 \mathrm{~K}$ operation. We speculate that this reduction in breakdown rates is correlated to the increased hardness of copper at cryogenic temperature. This reduction and the observed increase in the slopes of the breakdown lines at $77 \mathrm{~K}$ is in agreement with the previously observed behavior for accelerating cavities built with hard versus soft copper $[14,15]$. We should mention that the measured dark-current level in our cryogenic experiment was around $5 \mathrm{~mA}$ at the highest measured gradient. This results in a worst-case reduction in the rf power of only $0.2 \mathrm{MW}$, which is negligible compared to the steady-state rf power level in our measurements (15-30 MW).

The breakdown results are also in agreement with the reduced breakdown rates reported in [22]. Our results provide comprehensive breakdown statistics for the highgradient operation of NC accelerators at cryogenic temperature. These results give a more realistic intuition on the dependence of breakdown rates on the operating temperature compared to the study in [21] that showed no temperature dependence of the breakdown rates in the tested cavities. This reduction in breakdown rates at lower temperatures of operation is also predicted using different proposed breakdown models for normal conducting accelerating structures [8-11].

\section{CONCLUSION}

The presented experiment provides the first demonstration of the high-gradient acceleration of an electron beam at a cryogenic temperature of $77 \mathrm{~K}$. We reached a gradient level of $150 \mathrm{MV} / \mathrm{m}$ with a peak surface electric field of $375 \mathrm{MV} / \mathrm{m}$. The structure achieves a shunt impedance of $349 \mathrm{M} \Omega / \mathrm{m}$ and $\times 2.25$ enhancement in the beam loading capabilities compared to $300 \mathrm{~K}$ operation. The experiment provides a practical and cost-effective approach for the high-gradient operation of $\mathrm{NC}$ accelerators at cryogenic temperatures. We presented a comprehensive breakdown study at cryogenic temperature and high-gradient operation showing $\times \frac{1}{100}$ reductions in breakdown rates from 300 to $77 \mathrm{~K}$. The reduced breakdown rates agree with our understanding of the correlation between reduced breakdown rates and the increased material hardness. This experimental investigation provides a critical milestone for the practical use of $\mathrm{NC}$ accelerating systems at cryogenic temperatures which motivates many proposals for future discovery machines with optimized cost and performance parameters $[35,36]$.

\section{ACKNOWLEDGMENTS}

This research is supported by the U.S. Department of Energy, Contract No. DE-AC02-76SF00515.

[1] C. Adolphsen, The International Linear Collider technical design report-Volume 3. II: Accelerator baseline design, Thomas Jefferson and ANL Technical Report, Thomas Jefferson, 2013, https://arxiv.org/pdf/1306.6328.pdf.

[2] T. K. Charles, P. Giansiracusa, T. Lucas, R. Rassool, M. Volpi, C. Balazs, K. Afanaciev, V. Makarenko, A. Patapenka et al. (The CLIC, CLICdp Collaborations), arXiv:1812.06018.

[3] B. W. McNeil and N. R. Thompson, Nat. Photonics 4, 814 (2010).

[4] K. Kokurewicz, E. Brunetti, G. H. Welsh, S. Wiggins, M. Boyd, A. Sorensen, A. Chalmers, G. Schettino, A. Subiel, C. DesRosiers et al., Sci. Rep. 9, 10837 (2019).

[5] P. G. Maxim, S. G. Tantawi, and B. W. Loo, Jr., Radiotherapy Oncol. 139, 28 (2019).

[6] N. Solyak, AIP Conf. Proc. 1086, 365 (2009).

[7] H. Timko, F. Djurabekova, K. Nordlund, L. Costelle, K. Matyash, R. Schneider, A. Toerklep, G. Arnau-Izquierdo, A. Descoeudres, S. Calatroni, M. Taborelli, and W. Wuensch, Phys. Rev. B 81, 184109 (2010).

[8] A. Grudiev, S. Calatroni, and W. Wuensch, Phys. Rev. ST Accel. Beams 12, 102001 (2009).

[9] K. Nordlund and F. Djurabekova, Phys. Rev. ST Accel. Beams 15, 071002 (2012).

[10] V. A. Dolgashev, in Proceedings of the 2003 Particle Accelerator Conference, Portland, OR (IEEE, New York, 2003), Vol. 2, pp. 1267-1269, https://ieeexplore.ieee.org/ document/1289674.

[11] L. Laurent, S. Tantawi, V. Dolgashev, C. Nantista, Y. Higashi, M. Aicheler, S. Heikkinen, and W. Wuensch, Phys. Rev. ST Accel. Beams 14, 041001 (2011).

[12] E. Z. Engelberg, Y. Ashkenazy, and M. Assaf, Phys. Rev. Lett. 120, 124801 (2018).

[13] E. Z. Engelberg, A. B. Yashar, Y. Ashkenazy, M. Assaf, and I. Popov, Phys. Rev. Accel. Beams 22, 083501 (2019).

[14] V. A. Dolgashev, AIP Conf. Proc. 1507, 76 (2012). 
[15] V. Dolgashev et al., in the 2nd European Advanced Accelerator Concepts Workshop (EAAC 2015), La Biodola, Isola d'Elba, Italy (2015), pp. 13-19, https:// agenda.infn.it/event/8146/contributions/71603/attachments/ 51963/61378/Dolgashev_EAAC2012_High_gradient_ metallic_structures_final_ 14Sep2015.pdf.

[16] V. Dolgashev and S. Tantawi, Design of high efficiency high power electron accelerator systems based on normal conducting rf technology for energy and environmental applications, SLAC Technical Report, 2018.

[17] R. P. Reed and R. P. Mikesell, U.S. Department of Commerce, National Bureau of Standards, 1967.

[18] A. McEuen, P. Lui, E. Tanabe, and V. Vaguine, IEEE Trans. Nucl. Sci. 32, 2972 (1985).

[19] A. Saversky and I. Shchedrin, in Proceedings of the International Conference on Particle Accelerators (IEEE, New York, 1993), pp. 1030-1032.

[20] A. Iino, S. Yamaguchi, T. Higo, S. Michizono, T. Shintomi, Y. Funahashi, S. Matsumoto, J. Endo, and K. Ueno, Highpower test of $\mathrm{C}$-band accelerating structure at $20 \mathrm{~K}$, Proceedings of the 13th annual meeting of Particle Accelerator Society of Japan (2016), http://www.pasj.jp/ web_publish/pasj2016/proceedings/PDF/TUP0/TUP018 .pdf.

[21] H. H. Braun, S. Döbert, I. Wilson, and W. Wuensch, Phys. Rev. Lett. 90, 224801 (2003).

[22] A. Cahill, J. Rosenzweig, V. A. Dolgashev, S. G. Tantawi, and S. Weathersby, Phys. Rev. Accel. Beams 21, 102002 (2018).
[23] A. Cahill, J. Rosenzweig, V. Dolgashev, Z. Li, S. Tantawi, and S. Weathersby, Phys. Rev. Accel. Beams 21, 061301 (2018).

[24] S. Tantawi, M. Nasr, Z. Li, C. Limborg, and P. Borchard, Phys. Rev. Accel. Beams 23, 092001 (2020).

[25] G. Reuter and E. Sondheimer, Proc. R. Soc. A 195, 336 (1948).

[26] R. A. Matula, J. Phys. Chem. Ref. Data 8, 1147 (1979).

[27] C. Kittel and P. McEuen, Introduction to Solid State Physics (Wiley, New York, 1976), Vol. 8.

[28] G. Stupakov, K. Bane, P. Emma, and B. Podobedov, Phys. Rev. ST Accel. Beams 18, 034402 (2015).

[29] C. Limborg-Deprey, C. Adolphsen, S. Chu, M. Dunning, K. Jobe, E. Jongewaard, and C. Hast, An X-band gun test area at SLAC, SLAC Technical Report No. SLAC-PUB14692, 2011.

[30] J. Wang, Accelerator structure development for NLC/GLC, SLAC Technical Report No. SLAC-PUB-10370, 2004.

[31] S. G. Tantawi, C. D. Nantista, V. A. Dolgashev, C. Pearson, J. Nelson, K. Jobe, J. Chan, K. Fant, J. Frisch, and D. Atkinson, Phys. Rev. ST Accel. Beams 8, 042002 (2005).

[32] F. Nix and D. MacNair, Phys. Rev. 60, 597 (1941).

[33] D. Kajfez, IEEE Trans. Microwave Theory Tech. 42, 1149 (1994).

[34] T. P. Wangler, RF Linear Accelerators (John Wiley \& Sons, New York, 2008), Chap. 5.

[35] K. L. Bane, T. L. Barklow, M. Breidenbach, C. P. Burkhart, E. A. Fauve, A. R. Gold, V. Heloin, Z. Li, E. A. Nanni, M. Nasr et al., arXiv:1807.10195.

[36] J. Rosenzweig, N. Majernik, R. Robles, G. Andonian, O. Camacho, A. Fukasawa, A. Kogar, G. Lawler, J. Miao, P. Musumeci et al., New J. Phys. 22, 093067 (2020). 